\title{
Acodazole Hydrochloride
}

National Cancer Institute

\section{Source}

National Cancer Institute. Acodazole Hydrochloride. NCI Thesaurus. Code C71627.

The hydrochloride salt of acodazole, a synthetic imidazoquinoline with antineoplastic activity. Acodazole intercalates into DNA, resulting in disruption of DNA replication. Use of this agent has been associated with significant cardiotoxicity. 